\title{
Macular Optical Coherence Tomography Angiography in Nephropathic Patients with Diabetic Retinopathy in Iran: A Prospective Case-Control Study
}

\author{
Ali Ahmadzadeh Amiri • Majid Reza Sheikh Rezaee • Amir Ahmadzadeh Amiri • \\ Tayebeh Soleymanian · Reza Jafari · Ahmad Ahmadzadeh Amiri
}

Received: December 29, 2019 / Published online: February 19, 2020

(C) The Author(s) 2020

\begin{abstract}
Background: Diabetic macular ischemia (DMI) is an important category of diabetic retinopathy (DR) which leads to severe visual loss. Clinically, it is defined by an enlargement of the foveal avascular zone (FAZ) that can be detected by optical coherence tomography angiography (OCTA). Studies have described a relationship between renal disease and these changes in FAZ area. The aim of this study was to compare disturbances in FAZ area in diabetic patients with or without overt nephropathy.
\end{abstract}

Enhanced Digital Features To view enhanced digital features for this article go to https://doi.org/10.6084/ m9.figshare.11792043.

A. Ahmadzadeh Amiri $(\varangle) \cdot$ A. Ahmadzadeh Amiri Tehran University of Medical Sciences, Tehran, Iran e-mail: ali_ahmdzdh@yahoo.com

M. R. Sheikh Rezaee · R. Jafari · A. Ahmadzadeh Amiri

Department of Ophthalmology, Bu Ali Sina

Hospital, Mazandaran University of Medical

Sciences, Sari, Iran

T. Soleymanian

Department of Nephrology, Shariati Hospital,

Tehran University of Medical Sciences, Tehran, Iran
Methods: Following approval of the ethics committee, we examined diabetic patients with retinopathy. Patients were divided into two groups of DR, namely, with overt nephropathy and without overt nephropathy. The FAZ area was measured using OCTA. A $P$ value of $<0.05$ was considered to be statistically significant.

Result: A total of 46 patients (78 eyes) were enrolled in this study. All eyes with DR showed significant changes in FAZ area, but the sizes of the FAZ area were larger in both the superficial and deep layers in patients with clinical albuminuria than in those with no microalbuminuria $(P=0.007$ and $P=0.002$, respectively).

Conclusion: These results demonstrate that OCTA provides highly detailed information on retinal microvasculature and that it is a reliable modality to assess DR progression in patients with nephropathy. They also show that renal impairment as a systemic risk factor was associated with enlarged FAZ area in DM.

Keywords: Angiography;

Diabetic nephropathies; Diabetic retinopathy; Macular ischemia; Optical coherence; Tomography 


\section{Key Summary Points}

\section{Why carry out this study?}

Diabetic retinopathy (DR) is one of the most important complications of diabetes in a country like Iran which has a high incidence of type 2 diabetes, and it can place a heavy economic burden to the healthcare system. Diabetic macular ischemia (DMI), a category of DR, can cause severe visual loss.

Clinically, DMI can be defined by microvascular nonperfusion in the macular region that can be detected by optical coherence tomography angiography (OCTA).

Our aim was to assess whether diabetic nephropathy as an indication of diabetic severity is correlated with increased macular nonperfusion.

\section{What was learned from the study?}

In patients with retinopathy, the surface area of the foveal avascular zone was significantly larger in those with diabetic nephropathy than in those without diabetic nephropathy.

Patients with diabetic nephropathy are more susceptible to DMI.

\section{INTRODUCTION}

Diabetes mellitus is a global health concern, affecting more than 422 million people worldwide according to the most recent World Health Organization Global Report on Diabetes [1]. Approximately $25 \%$ of people with DM have some degree of diabetic retinopathy (DR) [2]. Visual loss in people with diabetes is mostly caused by three etiologies: diabetic macular edema (DME), complications of retinal neovascularization (vitreous hemorrhage and retinal detachment), and diabetic macular ischemia (DMI) $[3,4]$.

DMI is an important category of DR which leads to severe visual loss. It is characterized by occlusion and atrophy of the retinal capillaries in the macula, accompanied by the narrowing or obliteration of precapillary arterioles, and it affects approximately $7 \%$ of patients with DR [3]. Clinically, it is defined by an enlargement of the foveal avascular zone (FAZ) and paramacular areas of the capillary dropout [5]. The retinal vasculature is mainly comprised of two interconnected capillary plexuses: the superficial capillary plexus (SCP) and deep capillary plexus (DCP). The DCP is probably the most important provider of blood supply to the central macula, including the photoreceptors [6].

The gold standard modality for the measurement of retinal microvasculature remains fluorescein angiography (FA). However, this diagnostic procedure has two major disadvantages: it is a time-consuming and invasive modality that requires venipuncture and dye infusion, and it only provides two-dimensional images $[5,6]$. A recently developed noninvasive technology using optical coherence tomography angiography (OCTA) enables a faster acquisition of retinal vasculature via motion contrast imaging and provides highly detailed three-dimensional images without the need for any contrast fluid $[5,7,8]$. Studies of diabetic patients using OCTA indicated enlargement and irregularity of the vascular arcades of the FAZ and visible areas of reduced capillary density $[9,10]$.

Studies have shown that the presence of structural changes in the retinal microvasculature is associated with diabetic nephropathy (DN) and decreased renal function [11-13]. Diabetic patients with either micro- or clinical albuminuria frequently present with visionthreatening DR [14].

Although previous studies using OCTA have described abnormalities in the FAZ in patients with various retinal diseases, these studies did not provide information on the systemic conditions that may confound the vascular perfusion $[15,16]$. Therefore, the aim of this study was to assess the macular microvascular abnormality using OCTA by measuring the FAZ area 
as an indication for the diagnosis and quantification of DMI in patients with DR with or without overt nephropathy.

\section{METHODS}

This prospective case-control study was performed between 1 February 2018 and 30 August 2018. Patients with type 2 diabetes for $>$ 10 years who had DR were included. These patients ranged in age from 30 to 70 years. Exclusion criteria were: poorly controlled hypertension [systolic blood pressure (SBP) $\geq 180 \mathrm{mmHg}$ or diastolic blood pressure (DBP) $\geq 110 \mathrm{mmHg}$; pregnancy or breastfeeding; history of vitreoretinal surgery; history of macular grid lasering; media opacities obscuring retinal imaging and/or obstacle visualization of the FAZ; epimacular membrane; macular hole; underlying systemic diseases, such as rheumatologic disorders and malignancies, treated or not with chemotherapy.

The patients were divided into two groups, namely, those with overt nephropathy and those without overt nephropathy, based on the presence or absence of gross albuminuria ( $\geq 300 \mathrm{mg} / \mathrm{L}$ per $24 \mathrm{~h}$ ). Data on age, sex, height, weight, duration of diabetes, the presence of hypertension, and laboratory test results, including glycated hemoglobin (HbA1c), serum albumin, and creatinine, were also collected.

Diabetic retinopathy was graded according to the International Diabetic Retinopathy Severity Scale by experienced ophthalmologists $[17,18]$.

Urine albumin was measured by 24-h urine collection. Serum creatinine (Scr) was standardized with calibration traceable to an isotope-dilution mass spectrometry reference measurement procedure, and the estimated glomerular filtration rate (eGFR) was calculated by the Modification of Diet in Renal Disease (MDRD) equation $\left(175 \times \mathrm{Scr}^{-1.154} \times \mathrm{age}^{-0.203}\right.$ [× 0.742, if female]).

Comprehensive ophthalmologic examinations were performed. These included best-corrected visual acuity (BCVA) measurements using a standard vision chart, with the results recorded as the logarithmic minimum angle of resolution (LogMAR); slit-lamp biomicroscopy; spectral-domain optical coherence tomography (OCT) for retinal nerve fiber layer (RNFL) thickness; average thickness within the inner ring defined as the central foveal subfield (CSF) thickness assessment; and DME. A full and precise dilated fundus examination was performed, and OCTA results were used to assess macular nonperfusion.

The superficial, deep, and full retina FAZ areas in the macular region were evaluated (expressed in $\mathrm{mm}^{2}$ ). The superficial FAZ area is located $3 \mu \mathrm{m}$ beneath the internal limiting membrane and the outer boundary is located at $15 \mu \mathrm{m}$ beneath the inner plexiform layer. The image of the deep FAZ area was segmented with an inner boundary $15 \mu \mathrm{m}$ beneath the inner plexiform layer and an outer boundary at $70 \mu \mathrm{m}$ beneath the inner plexiform layer (OCTA view of SCP, DCP, and full retinal layers are shown in Fig. 1). The scanning area was captured in enface $3 \times 3-\mathrm{mm}$ image sections. The FAZ was measured using the caliper provided in the AngioPlex OCT angiography system (Carl Zeiss AG, Oberkochen, Germany) by two experienced examiners. The diameter of the FAZ was measured from the innermost visible well-defined vascular marking from one end to the other both horizontally and vertically. The quantification of macular nonperfusion in OCTA captured images was completed using ImageJ 1.48 software.

Statistical analyses were performed using PASW statistics software version 18.0.0 (IBM SPSS Statistics, Armonk, NY, USA). Numbers with percentages and means with standard deviations (SD) were used to express the qualitative and quantitative variables, respectively. Nonparametric analysis of variance for comparing the two groups was used alongside other nonparametric tests. Qualitative variables were compared by the Fisher exact test. $P$ value $<0.05$ was considered to be statistically significant.

Approval of this study was granted prior to patient enrollment by the Mazandaran University of Medical Sciences ethics committee (ID: IR.MAZUMS.REC.1398.299). This study was performed according to the tenets of the Declaration of Helsinki of 1964, as revised in 2013. 

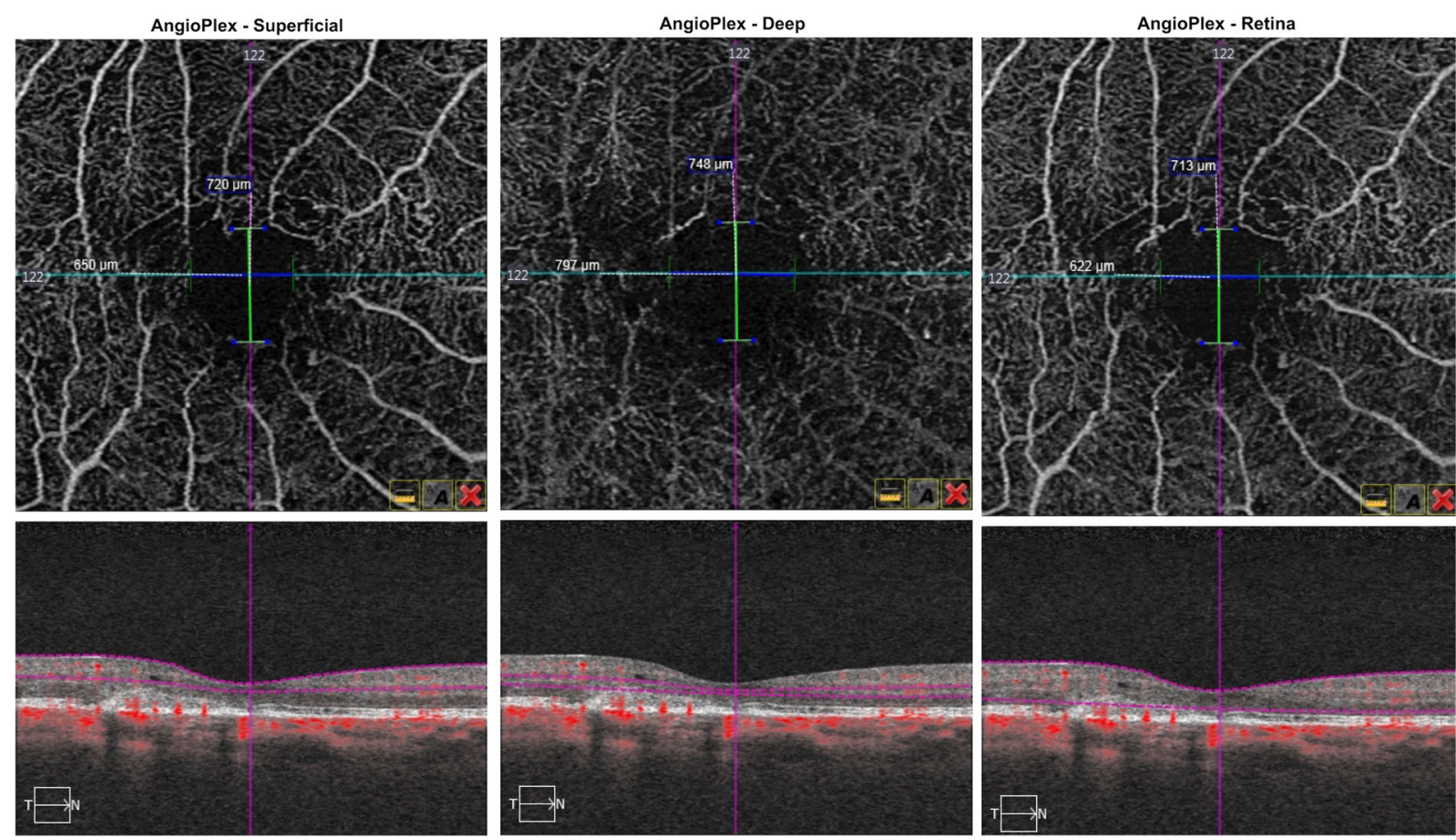

Fig. 1 Optical coherence tomography angiography (OCTA) images of superficial, deep, and full retina foveal avascular zone (FAZ) areas of eye of a patient with nonproliferative diabetic retinopathy and nephropathy. Left: Capillary scan of a right macula center. $3 \times 3-\mathrm{mm}$ superficial retinal layer flow image and 3-mm horizontal B-scan through fovea with segmentation lines outlining the superficial retinal layer. Center: $3 \times 3$-mm-deep retinal layer flow image and 3-mm horizontal B-scan through

All patients who met the inclusion criteria provided written informed consent before enrollment.

\section{RESULTS}

A total of 46 patients (78 eyes) were enrolled in this study, of whom 22 had overt nephropathy and 24 were without overt nephropathy. The mean ( \pm SD) age of these patients was 58.9 \pm 11.4 years, and the mean $\mathrm{HbA} 1 \mathrm{c}$ level was 7.9 $\pm 1.8 \%$.

The baseline demographics and clinical characteristics of the enrolled patients are given in Table 1 . There were no statistically significant differences in terms of demographic characteristics between the two groups. However, fovea with segmentation lines outlining the deep retinal layer. Right: $3 \times 3-\mathrm{mm}$ total retinal layer flow image and 3-mm horizontal B-scan through fovea with segmentation lines outlining the total retinal layer. The FAZ appears to be enlarged, with the presence of perifoveal capillary dropout. Caliper measurement of both the horizontal and vertical diameters of the superficial, deep capillary plexus, and total retina areas are illustrated

patients with overt nephropathy had significantly higher SBP and DBP $(P=0.001$ and $P=0.005$, respectively). Table 1 also indicates that a higher level of proteinuria in nephropathic patients compared to those without overt nephropathy $(6501 \pm 3015 \quad$ vs. $273 \pm 183 \mathrm{mg} / 24 \mathrm{~h}$ ) could yield reasonable information regarding significantly lower serum albumin in the former compared with the latter $(2.96 \pm 0.87$ vs. $3.75 \pm 0.55 \mathrm{~g} / \mathrm{dL})$.

The ocular characteristics of the two groups are summarized in Table 2 . With the exception of central subfield (CSF), there were no significant differences between patients with overt nephropathy and those without overt nephropathy in terms of the results of the different ophthalmologic examinations. Patients with overt nephropathy were found to have a 
Table 1 Demographic and clinical characteristics of patients enrolled in the study

\begin{tabular}{llll}
\hline Variables & $\begin{array}{l}\text { DR with overt nephropathy } \\
(\boldsymbol{n}=\mathbf{2 2} \text { patients })\end{array}$ & $\begin{array}{l}\text { DR without overt nephropathy } \\
(\boldsymbol{n}=\mathbf{2 4} \text { patients })\end{array}$ & $\boldsymbol{P}$ value \\
\hline Demographic variables & & $58.25 \pm 9.74$ & 0.590 \\
Age (years) & $59.81 \pm 9.84$ & $13 / 11$ & 0.607 \\
Sex (male/female) & $12 / 10$ & $25.58 \pm 2.73$ & 0.754 \\
BMI $\left(\mathrm{kg} / \mathrm{m}^{2}\right)$ & $25.82 \pm 2.46$ & & 0.001 \\
Clinical and laboratory variables & & $137.08 \pm 14.06$ & 0.005 \\
Systolic blood pressure (mmHg) & $148.64 \pm 5.39$ & $80.83 \pm 7.02$ & 0.458 \\
Diastolic blood pressure (mmHg) & $86.14 \pm 4.86$ & $22.96 \pm 3.69$ & 0.468 \\
Duration of diabetes (years) & $23.77 \pm 3.68$ & $7.96 \pm 1.03$ & 0.077 \\
HbAlc level $(\%)$ & $8.19 \pm 1.08$ & $48.29 \pm 8.96$ & 0.093 \\
Estimated GFR (mL/min) & $44.27 \pm 5.52$ & $1.68 \pm 0.42$ & $<0.001$ \\
Creatinine $(\mathrm{mg} / \mathrm{dL})$ & $1.86 \pm 0.27$ & $3.75 \pm 0.55$ & $<0.001$ \\
Serum albumin $(\mathrm{g} / \mathrm{dL})$ & $2.96 \pm 0.87$ & $273 \pm 183$ & \\
Urine albumin $(\mathrm{mg} / 24 \mathrm{~h})$ & $6501 \pm 3015$ & & \\
\hline
\end{tabular}

$B M I$ Body mass index, $D R$ diabetic retinopathy, eGFR glomerular filtration rate, HbAlc glycosylated hemoglobin

significantly thicker CSF than with patients without nephropathy $(P=0.036)$.

The nonperfusion status of the FAZ area on the OCTA images in DR is given in Table 3. In all eyes, the full retinal FAZ sizes were larger in the eyes of patients with overt nephropathy than in those without $(P=0.001)$. In addition, the mean FAZ area on OCTA angiogram images at the level of both the SCP and DCP was significantly larger in patients with overt nephropathy than in those without $(P=0.007$ and $P=0.002$, respectively) (Fig. 2).

The correlation between either mean SBP or DBP and FAZ size was not statistically significant among groups (FAZ surface and mean SBP, $P=0.903 ; \quad$ FAZ deep; $P=0.990 ; \quad$ FAZ full, $P=0.963 ; \quad F A Z$ surface and mean DBP, $P=0.978 ; \quad$ FAZ deep, $P=0.796 ; \quad$ FAZ full, $P=0.824)$.

The correlation between mean serum albumin and FAZ size was also not statistically significant among groups (FAZ surface, $P=0.270$; FAZ deep, $P=0.415$; FAZ full, $P=0.426$ ).

\section{DISCUSSION}

As mentioned earlier, in recent years FA has been utilized to assess DMI. FA can reveal the vascular mapping of the retina as well as the leakages caused by microaneurysms; therefore, it remains the gold standard modality for the diagnosis and management of DR. Nevertheless, the invasiveness and probable side effects of fluorescein injection always have to be taken into consideration, especially in patients with nephropathy [15]. A well-known feature of DR in FA is a significant enlargement of FAZ size $[19,20]$. OCTA is a noninvasive diagnostic method that can provide information about retinal capillary microvasculature within specific layers. This modality has shown a wide range of clinical applications and proved to be a suitable diagnosing alternative for many eye conditions, such as glaucoma, exudative agerelated macular degeneration, as well as DR [15].

$\mathrm{DN}$, as the primary cause of chronic kidney disease, leads to $40 \%$ of all new cases of endstage renal disease annually. Overt nephropathy 
Table 2 Ocular characteristics of patients enrolled in the study

\begin{tabular}{llll}
\hline Variables & $\begin{array}{l}\text { DR with overt nephropathy } \\
(\boldsymbol{n}=\mathbf{2 2} \text { patients })\end{array}$ & $\begin{array}{l}\text { DR without overt nephropathy } \\
(\boldsymbol{n}=\mathbf{2 4} \text { patients })\end{array}$ & $\boldsymbol{P}$ value \\
\hline Ocular examination & & & 0.360 \\
Eyes examined, $n$ (right/left) & $20 / 19$ & $18 / 21$ & 0.116 \\
BCVA (LogMAR) & $0.44 \pm 0.29$ & $0.31 \pm 0.25$ & 0.810 \\
IOP (mmHg) & $15.18 \pm 0.82$ & $15.25 \pm 1.06$ & 0.710 \\
RNFL thickness $(\mu \mathrm{m})$ & $87.23 \pm 7.96$ & $88.19 \pm 9.33$ & 0.036 \\
CSF thickness $(\mu \mathrm{m})$ & $289.73 \pm 7.63$ & $284.21 \pm 9.51$ & 0.905 \\
DR severity (number of eyes) & & & \\
NPDR & 23 & 24 & \\
PDR, & 16 & 15 & \\
No DME & 5 & 9 & \\
DME & 34 & 30 & \\
\hline
\end{tabular}

$B C V A$ best corrected visual acuity, $C S F$ central subfield, $D M E$ diabetic macular edema, IOP intraocular pressure, $\log M A R$ logarithmic minimum angle of resolution, NPDR nonproliferative diabetic retinopathy, $P D R$ proliferative diabetic retinopathy, $R N F L$ retinal nerve fiber layer

presents with clinical albuminuria, progressive reduction in GFR, and increases in blood pressure [21]. In addition, diabetic patients with clinical albuminuria are at risk of developing vision-threatening DR $[14,22]$.

In this case-control study, we described the application of OCTA to study patients with DR and examine the effect of nephropathy as a systemic metabolic and vascular risk factor. We observed that the FAZ parameters of OCTA among diabetic patients were significantly larger in patients with overt nephropathy than in those without overt nephropathy. To our knowledge, this is the first study to investigate the effect of nephropathy as an indication of diabetes severity on changes in FAZ area in patients with DR.

Our findings could explain the observed functional and structural changes in the retinal microvasculature, demonstrating that patients with overt nephropathy carry an elevated risk of DMI, which can be explained by macular capillary nonperfusion in the presence of clinical albuminuria. In this study, we performed a detailed quantitative analysis of the FAZ surface to assess foveal perfusion disturbance in patients with overt nephropathy using OCTA. A statistically significant difference was observed between the two patient groups (those with overt nephropathy and those without) for each FAZ surface parameter (superficial, deep, and full retina). These results are in agreement with those of other studies which evaluated the reliability of FAZ measurements using OCTA as a marker of diabetic microvascular changes [23-26]. Similar to the pathogenesis of DMI, the progression of nephropathy is ultimately associated with capillary nonperfusion, which leads to podocyte death, neoangiogenesis of the glomerular capillaries, and loss of capillaries in the glomerulus and interstitium. Therefore, our results emphasize the pivotal role that endothelial hypoxia plays in decreasing the GFR in patients with $\mathrm{DN}[27,28]$.

As in previous studies, we found that eyes with DR and DME had increased FAZ area and reduced vessel density, as shown in the OCTA images, compared to those with DR without DME $[9,29]$. Also, the OCTA images showed that cystic spaces are surrounded by capillary 
Table 3 Foveal avascular zone area as assessed by optical coherence tomography angiography

\begin{tabular}{llll}
\hline FAZ measures $\left(\mathbf{m m}^{\mathbf{2}}\right)$ & DR with overt nephropathy & DR without overt nephropathy & $\boldsymbol{P}$ value \\
\hline Superficial retinal capillary plexus & $0.484 \pm 0.053$ & $0.446 \pm 0.036$ & 0.007 \\
Deep retinal capillary plexus & $0.498 \pm 0.053$ & $0.454 \pm 0.035$ & 0.002 \\
Full retina & $0.501 \pm 0.052$ & $0.456 \pm 0.036$ & 0.001 \\
\hline
\end{tabular}

Values in tables are presented as the mean \pm standard deviation

FAZ Foveal avascular zone

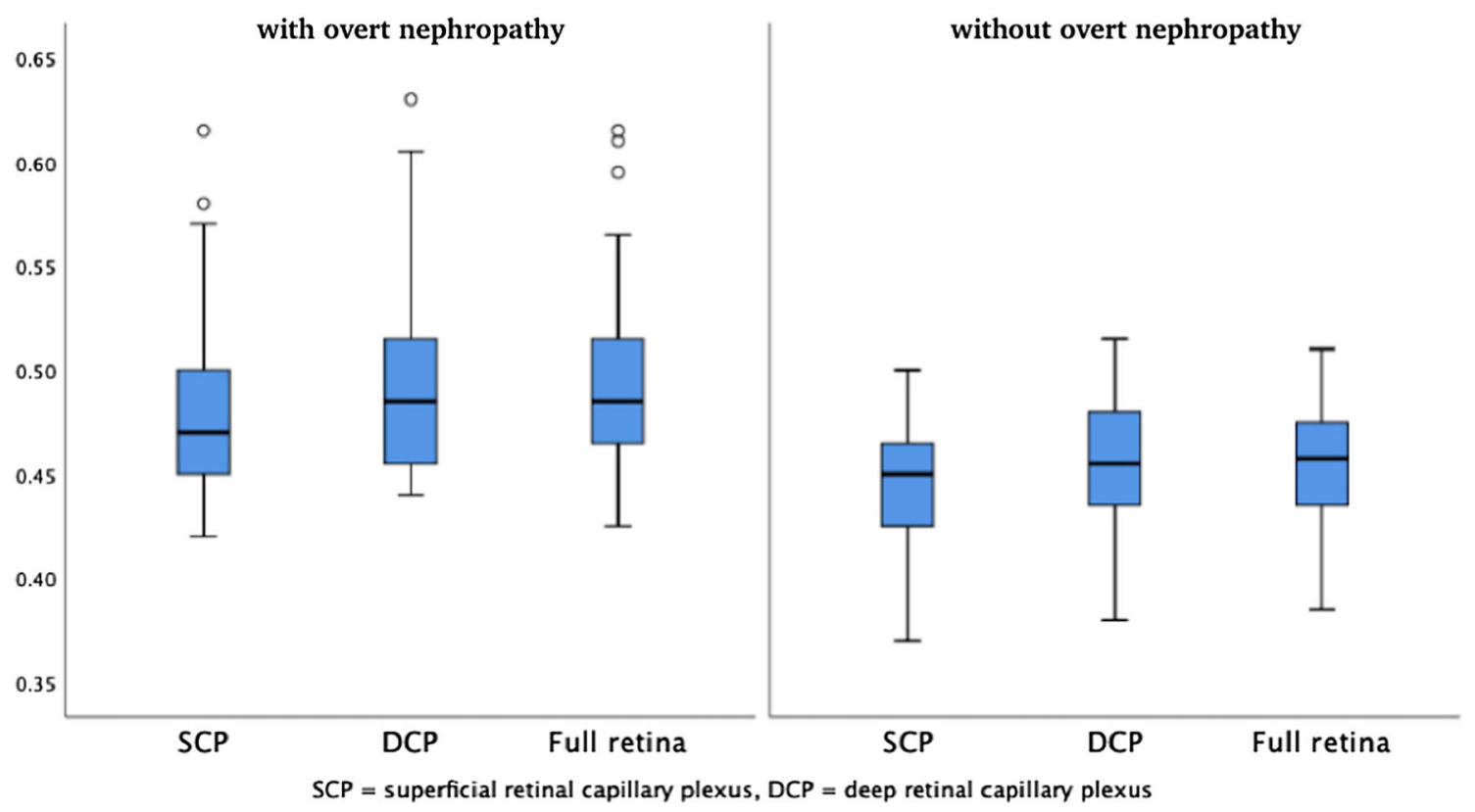

Fig. 2 Box plot showing FAZ area measurements using OCTA in patients with and without diabetic overt nephropathy. $D C P$ Deep retinal capillary plexus, $S C P$ superficial retinal capillary plexus

nonperfusion, which shows no evidence of reperfusion after the resolution of DME, suggesting that DME might preferentially develop in areas of ischemia [30]. Based on the results of other studies, the development of DR but not DME was correlated with a reduction in GFR [31]. Jeng et al. illustrated that the incidences of nonproliferative diabetic retinopathy, proliferative diabetic retinopathy, and DME increased with time in patients with $\mathrm{DN}$, who also presented a higher rate of DR development than did patients without DN [32].

Regarding blood pressure, our results show that patients with higher blood pressure had a significant enlargement of FAZ size compared with patients with lower blood pressure. This result is partly consistent with the findings reported in earlier studies indicating that increased blood pressure is a major risk factor for DR progression [33]. The most interesting result obtained in our study was that the frequency of DME development did not differ between patients in the two different patient groups regardless of blood pressure status. However, patients with significantly higher blood pressure still showed an increased risk of developing perifoveal capillary dropout compared to patients with lower blood pressure. Although a number of studies have suggested that hypertension is associated with DR, some 
longitudinal data, such as data from the United Kingdom Prospective Diabetes Study (UKPDS) and Wisconsin Epidemiologic Study of Diabetic Retinopathy (WESDR), have still been inconsistent [34-37]. Nonetheless, no association has been found for the presence or absence of hypertension and DR in patients with type 2 diabetes [37]. It has been postulated that hypertension independent of hyperglycemia affects DR through impaired retinal autoregulation, which in turn upregulates the expression of vascular endothelial growth factor (VEGF) in retinal endothelial cells and leads to progressive alterations in the retinal microvasculature, vasopermeability, and areas of retinal occlusion [38].

There are a number of limitations to this study, including the small sample size, the study design (OCTA was not compared with FA), and the issue of the automated preset in the software of the AngioPlex OCT angiography system with its limited computing of OCTA imaging. Despite the small sample size, we only included eyes with DR which previously had not received any sort of treatment. Therefore, any changes in microvasculature in eyes that had been formerly treated and possibly not to be due to the natural course of the disease were not included in the analysis and would not affect the OCTA parameters. This exclusion of previously treated eyes can be regarded as a strength of this study. Furthermore, since the injection of dye is nephrotoxic, the significance of a comparison between the OCTA FAZ area FA results could be considered arguable. Although our findings have been fully validated both qualitatively and quantitatively with careful measures and statistical models, additional studies are needed to improve clinical practice and reduce the indications for the use of FA.

\section{CONCLUSION}

The results of this study show that OCTA may provide more detailed images than FA to explore both SCP and DCP in DR. This noninvasive technique offers improved quantification of the FAZ surface in diabetic patients as observed during progression of DR. All patients with DR showed significant changes in FAZ area on OCTA images, and these changes were especially more prominent in eyes of patients with overt nephropathy.

Different etiologies tend to exhibit similar trends in ocular parameter changes in DR. However, the process of excess body protein loss and metabolic waste may affect retinal microcirculation and cause more impaired macular perfusion that can be measured by OCTA and detected as FAZ size measurement. More importantly, we showed that renal impairment as a systemic metabolic and vascular risk factor was associated with enlarged FAZ size in patients with DM.

\section{ACKNOWLEDGEMENTS}

The authors of this article would like to acknowledge the staff of the Sari Bu Ali Sina Hospital eye research development center, Sari $\mathrm{Bu}$ Ali Sina Hospital medical laboratory, and Sari Tabarestan Eye Center for their cooperation. The authors would like to thank all participants of the study for their contribution.

Funding. Mazandaran University of Medical Sciences provided funding for the study. No funding or sponsorship was received for the publication of this article.

Authorship. All named authors meet the International Committee of Medical Journal Editors (ICMJE) criteria for authorship for this article, take responsibility for the integrity of the work as a whole, and have given their approval for this version to be published.

Disclosures. Ali Ahmadzadeh Amiri, Majid Reza Sheikh Rezaee, Amir Ahmadzadeh Amiri, Tayebeh Soleymanian, Reza Jafari, and Ahmad Ahmadzadeh Amiri report no potential conflict of interest relevant to this article.

Compliance with Ethics Guidelines. Approval of this study was granted prior to patient enrollment by the Mazandaran University of Medical Sciences ethics committee (ID: 
IR.MAZUMS.REC.1398.299). This study was performed according to the tenets of the Declaration of Helsinki of 1964, as revised in 2013. All patients who met the inclusion criteria provided written informed consent before enrollment.

Data Availability. The datasets generated during and/or analyzed during the current study are not publicly available due to the patient privacy principals of our research center but are available from the corresponding author on reasonable request and proper circumstances.

Open Access. This article is licensed under a Creative Commons Attribution-NonCommercial 4.0 International License, which permits any non-commercial use, sharing, adaptation, distribution and reproduction in any medium or format, as long as you give appropriate credit to the original author(s) and the source, provide a link to the Creative Commons licence, and indicate if changes were made. The images or other third party material in this article are included in the article's Creative Commons licence, unless indicated otherwise in a credit line to the material. If material is not included in the article's Creative Commons licence and your intended use is not permitted by statutory regulation or exceeds the permitted use, you will need to obtain permission directly from the copyright holder. To view a copy of this licence, visit http://creativecommons.org/licenses/bync/4.0/

\section{REFERENCES}

1. NCD Risk Factor Collaboration (NCD-RisC). Worldwide trends in diabetes since 1980: a pooled analysis of 751 population-based studies with 44 million participants. Lancet. 2016;387(10027): 1513-30.

2. Yau JW, Rogers SL, Kawasaki R, et al. Global prevalence and major risk factors of diabetic retinopathy. Diabetes Care. 2012;35(3):556-64.

3. Liew G, Sim DA, Keane PA, et al. Diabetic macular ischaemia is associated with narrower retinal arterioles in patients with type 2 diabetes. Acta Ophthalmol. 2015;93(1):e45-51.

4. Sayin N, Kara N, Pekel G. Ocular complications of diabetes mellitus. World J Diabetes. 2015;6(1): 92-108.

5. Garcia JM, Lima TT, Louzada RN, Rassi AT, Isaac DL, Avila M. Diabetic macular ischemia diagnosis: comparison between optical coherence tomography angiography and fluorescein angiography. J Ophthalmol. 2016;2016:3989310.

6. Campbell JP, Zhang M, Hwang TS, et al. Detailed vascular anatomy of the human retina by projection-resolved optical coherence tomography angiography. Sci Rep. 2017;7:42201.

7. Enders C, Baeuerle F, Lang GE, et al. Comparison between findings in optical coherence tomography angiography and in fluorescein angiography in patients with diabetic retinopathy. Ophthalmologica. 2020;243(1):21-6.

8. Hwang TS, Jia Y, Gao SS, et al. Optical coherence tomography angiography features of diabetic retinopathy. Retina. 2015;35(11):2371-6.

9. Di G, Weihong Y, Xiao Z, et al. A morphological study of the foveal avascular zone in patients with diabetes mellitus using optical coherence tomography angiography. Graefes Arch Clin Exp Ophthalmol. 2016;254(5):873-9.

10. Freiberg FJ, Pfau M, Wons J, Wirth MA, Becker MD, Michels S. Optical coherence tomography angiography of the foveal avascular zone in diabetic retinopathy. Graefes Arch Clin Exp Ophthalmol. 2016;254(6):1051-8.

11. Lee WJ, Sobrin L, Lee MJ, Kang MH, Seong M, Cho $H$. The relationship between diabetic retinopathy and diabetic nephropathy in a population-based study in Korea (KNHANES V-2, 3). Invest Ophthalmol Vis Sci. 2014;55(10):6547-53.

12. Lim LS, Cheung CY, Sabanayagam C, et al. Structural changes in the retinal microvasculature and renal function. Invest Ophthalmol Vis Sci. 2013;54(4):2970-6.

13. Edwards MS, Wilson DB, Craven TE, et al. Associations between retinal microvascular abnormalities and declining renal function in the elderly population: the Cardiovascular Health Study. Am J Kidney Dis. 2005;46(2):214-24.

14. Hammes HP, Welp R, Kempe HP, et al. Risk factors for retinopathy and DME in type 2 diabetes-results from the German/Austrian DPV database. PLoS ONE. 2015;10(7):e0132492. 
15. Chalam K, Sambhav K. Optical coherence tomography angiography in retinal diseases. J Ophthalmic Vis Res. 2016;11:84-92.

16. Falavarjani KG, Sarraf D. Optical coherence tomography angiography of the retina and choroid; current applications and future directions. J Curr Ophthalmol. 2017;29:1.

17. Wong TY, Sun J, Kawasaki R, et al. Guidelines on diabetic eye care: the international council of ophthalmology recommendations for screening, follow-up, referral, and treatment based on resource settings. Ophthalmology. 2018;125(10):1608-22.

18. Fong DS, Aiello LP, Ferris FL 3rd, Klein R. Diabetic retinopathy. Diabetes Care. 2004;27(10):2540-53.

19. Arend O, Wolf S, Remky A, Sponsel WE, Harris A, Bertram B, Reim M. Perifoveal microcirculation with noninsulin-dependent diabetes mellitus. Graefes Arch Clin Exp Ophthalmol. 1994;232: 225-31.

20. Mansour AM, Schachat A, Bodiford G, Haymond R. Foveal avascular zone in diabetes mellitus. Retina. 1993;13:125-8.

21. Skyler JS. Microvascular complications: retinopathy and nephropathy. Endocrinol Metab Clin North Am. 2001;30:833-56.

22. Boelter MC, Gross JL, Canani LH, et al. Proliferative diabetic retinopathy is associated with microalbuminuria in patients with type 2 diabetes. Braz J Med Biol Res. 2006;39(8):1033-9.

23. Gozlan J, Ingrand P, Lichtwitz $\mathrm{O}$, et al. Retinal microvascular alterations related to diabetes assessed by optical coherence tomography angiography: a cross-sectional analysis. Medicine (Baltimore). 2017;96(15):e6427.

24. Bradley PD, Sim DA, Keane PA, et al. The evaluation of diabetic macular ischemia using optical coherence tomography angiography. Invest Ophthalmol Vis Sci. 2016;57(2):626-31.

25. Nesper PL, Roberts PK, Onishi AC, et al. Quantifying microvascular abnormalities with increasing severity of diabetic retinopathy using optical coherence tomography angiography. Invest Ophthalmol Vis Sci. 2017;58(6):BIO307-BIO315.

26. Ting DSW, Tan GSW, Agrawal R, et al. Optical coherence tomographic angiography in type 2 diabetes and diabetic retinopathy. JAMA Ophthalmol. 2017;135(4):306-12.
27. Advani A, Gilbert RE. The endothelium in diabetic nephropathy. Semin Nephrol. 2012;32(2):199-207.

28. Arboleda-Velasquez JF, Valdez CN, Marko CK, D'Amore PA. From pathobiology to the targeting of pericytes for the treatment of diabetic retinopathy. Curr Diab Rep. 2015;15(2):573.

29. Kim AY, Chu Z, Shahidzadeh A, Wang RK, Puliafito CA, Kashani AH. Quantifying microvascular density and morphology in diabetic retinopathy using spectral-domain optical coherence tomography angiography. Investig Ophthalmol Vis Sci. 2016;57(9):ОСТ362-ОСТ370.

30. Mané V, Dupas B, Gaudric A, et al. Correlation between cystoid spaces in chronic diabetic macular edema and capillary nonperfusion detected by optical coherence tomography angiography. Retina. 2016;36[Suppl 1]:S102-S110110.

31. Man RE, Sasongko MB, Wang JJ, et al. The association of estimated glomerular filtration rate with diabetic retinopathy and macular edema. Invest Ophthalmol Vis Sci. 2015;56(8):4810-6.

32. Jeng CJ, Hsieh YT, Yang CM, Yang CH, Lin CL, Wang IJ. Diabetic retinopathy in patients with diabetic nephropathy: development and progression. PLoS ONE. 2016;11(8):e0161897.

33. Leske MC, Wu SY, Hennis A, et al. Hyperglycemia, blood pressure, and the 9-year incidence of diabetic retinopathy: the Barbados Eye Studies. Ophthalmology. 2005;112(5):799-805.

34. Norgaard K, Feldt-Rasmussen B, Deckert T. Is hypertension a major independent risk factor for retinopathy in type 1 diabetes? Diabet Med. 1991;8(4):334-7.

35. Schmittdiel JA, Steiner JF, Adams AS, et al. Diabetes care and outcomes for American Indians and Alaska natives in commercial integrated delivery systems: a Surveillance, PREvention, and ManagEment of Diabetes Mellitus SUPREME-DM Study. BMJ Open Diabetes Res Care. 2014;2(1):e000043.

36. Ballard DJ, Melton LJ 3rd, Dwyer MS, et al. Risk factors for diabetic retinopathy: a population-based study in Rochester. Minnesota. Diabetes Care. 1986;9(4):334-42.

37. Klein R, Klein BE, Moss SE. TheWisconsin epidemiological study of diabetic retinopathy: a review. Diabetes Metab Rev. 1989;5(7):559-70.

38. Srivastava BK, Rema M. Does hypertension play a role in diabetic retinopathy? J Assoc Phys India. 2005;53:803-8. 